\title{
BMJ Open Protocol for a prospective neuroimaging study investigating the supraspinal control of lower urinary tract function in healthy controls and patients with non-neurogenic lower urinary tract symptoms
}

\author{
Matthias Walter, ${ }^{1}$ Lars Michels, ${ }^{2}$ Spyros Kollias, ${ }^{2}$ Philip E van Kerrebroeck, ${ }^{3}$ \\ Thomas M Kessler, ${ }^{1}$ Ulrich Mehnert ${ }^{1}$
}

To cite: Walter M, Michels L, Kollias S, et al. Protocol for a prospective neuroimaging study investigating the supraspinal control of lower urinary tract function in healthy controls and patients with non-neurogenic lower urinary tract symptoms. BMJ Open 2014;4:e004357.

doi:10.1136/bmjopen-2013004357

- Prepublication history for this paper is available online. To view these files please visit the journal online (http://dx.doi.org/10.1136/ bmjopen-2013-004357).

Received 29 October 2013 Revised 23 April 2014 Accepted 29 April 2014

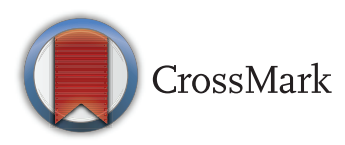

For numbered affiliations see end of article.

Correspondence to Dr Ulrich Mehnert; ulrich.mehnert@paralab. balgrist.ch

\section{ABSTRACT}

Introduction: Lower urinary tract symptoms (LUTS) are highly prevalent, cause an enormous economic burden on healthcare systems and significantly impair the quality of life $(\mathrm{Q} O \mathrm{~L})$ of affected patients. The dependence of the LUT on complex central neuronal circuits makes it unique in comparison to other visceral functions, such as the gastrointestinal tract, but also more vulnerable to neurological diseases.

Methods and analysis: This is a prospective neuroimaging study investigating the supraspinal control of LUT function in healthy controls and in patients with non-neurogenic LUTS. The clinical assessment will include medical history, neurourological examination, bladder diary, urine analysis, urodynamic investigations, as well as standardised questionnaires regarding LUTS and QoL. The acquisition of neuroimaging data will include structural assessments (T1-weighted imaging and diffusion tensor imaging) as well as functional investigations using blood-oxygen-level dependent sensitive functional MRI (fMRI) in a $3 \mathrm{~T}$ MR scanner. The fMRI will be performed during four different bladder tasks using an automated MR-compatible and MR-synchronised pump system. The first three task-related fMRIs will consist of automated, repetitive filling of $100 \mathrm{~mL}$ warm $\left(37^{\circ} \mathrm{C}\right)$ saline starting with (1) an empty bladder, (2) a low prefilled bladder volume $(100 \mathrm{~mL})$ and $(3)$ a high prefilled bladder volume (persistent desire to void). The fourth task-related fMRI will comprise of automated, repetitive filling of $100 \mathrm{~mL}$ cold $\left(4-8^{\circ} \mathrm{C}\right)$ saline starting with an empty bladder.

Ethics and dissemination: The local ethics committee approved this study (KEK-ZH-Nr. 20110346). The findings of the study will be published in peer-reviewed journals and presented at national and international scientific meetings.

Trial registration number: This study has been registered at clinicaltrials.gov (http://www.clinicaltrials. gov/ct2/show/NCT01768910).
Strengths and limitations of this study

- This will be the first study to identify brain networks of supraspinal LUT control in healthy subjects and abnormalities within such brain networks in patients with non-neurogenic LUTS using structural and functional MRI techniques in correlation with clinical measurements.

- Investigation of test-retest reliability has not yet been performed for neuroimaging of LUT tasks. However, this is important for the interpretation of participants' activations in regard to the validity of these activations, i.e. absolute and consistent agreement (ICC) from visit to visit.

- Comparison of clinical correlates of treatment efficacy in patients with non-neurogenic LUTS with the associated changes in brain activity and connectivity.

\section{INTRODUCTION}

Lower urinary tract symptoms (LUTS) are highly prevalent, that is, about $11 \%$ in the worldwide population in 2008 , and forecasted to increase up to $20 \%$ until $2018 .^{12}$ Moreover, LUTS cause an enormous economic burden on each healthcare system, ${ }^{34}$ which is comparable to diabetes mellitus, ${ }^{5}$ and significantly impair the quality of life (QoL) of affected patients. ${ }^{6} 7$

For proper functioning, LUT structures, that is, bladder, bladder neck, urethra and urethral sphincter, rely on intact neuronal innervations that are under the control of a complex supraspinal network. ${ }^{8-10}$ The dependence of the LUT on such complex central neuronal circuits makes it unique in comparison with other visceral functions, for example, gastrointestinal tract, but also more vulnerable to neurological diseases. ${ }^{10}$ 
Recent neuroimaging studies have shown that patients with neurological disorders such as Parkinson's disease $^{11-13}$ and spinal cord injury ${ }^{14}$ demonstrate different supraspinal activity patterns compared with healthy controls in response to LUT stimulation tasks, which might represent a neural correlate to their LUTS.

Although there are several concepts regarding the human LUT function and neuronal control in normal and pathological conditions, the exact pathophysiological mechanisms involved remain largely unknown. ${ }^{9}$ Despite the popularity of resting-state functional MRI (RS-fMRI) ${ }^{15-20}$ and diffusion tensor imaging (DTI) ${ }^{21}$ in other fields in neuroscience, these techniques have not been applied in the context of supraspinal LUT control. There are only two DTI studies published in regard to LUT control in general: (1) a case report by Théaudin $e t a l^{22}$ studying spinal cord infarctions and clinical symptoms and (2) a prospective study by van der Jagt $e t a t^{23}$ investigating architectural configuration and microstructural properties of the sacral plexus.

As cortical and subcortical (eg, brainstem) brain regions are crucial for voluntary LUT control, ${ }^{10} 2425$ investigation of the supraspinal regions with highresolution imaging techniques, such as fMRI, can significantly contribute to increase our understanding of the effects of supraspinal lesions and alterations related to LUTS. $^{10} 26$

In this study, we are aiming to identify supraspinal areas associated with LUT control in healthy controls and in patients with non-neurogenic LUTS. Task-related blood-oxygen-level dependent (BOLD) and RS-fMRI will be used along with structural MRI (T1-weighted MRI and DTI). Hence, we will examine whether bladder processing is already altered on the structural level and on baseline (RS-fMRI) functional connectivity (FC). For example, multiple repetition of the RS-fMRI will help to understand whether manipulation of sensory perception (induced by infusion and withdrawal) will alter the default mode network ${ }^{20}$ of the brain. Furthermore, we can examine volumetric parameters (eg, grey matter concentration) by voxel-based morphometry (VBM), ${ }^{27}$ structural integrity and connectivity of white matter tracts (DTI) as well as FC.

This unique and detailed multimodal imaging protocol should pinpoint to structural and functional processing units involved during supraspinal LUT control and should identify all dysfunctional neuronal components in patients with disturbed LUT control.

Importantly, we will investigate the reliability ${ }^{28}$ of BOLD signals in task-related fMRI and RS-fMRI in healthy controls and patients with non-neurogenic LUTS. The testretest validation, that is, the intraclass correlation coefficient (ICC) for absolute or consistent agreement of subject activations from visit to visit, has not been evaluated in regard to the supraspinal LUT control yet.

\section{METHODS AND ANALYSIS}

\section{Study design}

This prospective research study will be conducted at the University of Zürich, Zürich, Switzerland.

\section{Study population and recruitment}

According to the inclusion and exclusion criteria (table 1), we will investigate patients with non-neurogenic LUTS and healthy controls with an unimpaired LUT function.

Table 1 Inclusion and exclusion criteria for all participants

\begin{tabular}{ll}
\hline Groups & Inclusion criteria \\
\hline All participants & Right handed \\
& Gender (female and male) \\
MR suitability $18-55$ years \\
Written informed consent
\end{tabular}

$\begin{array}{ll}\text { Healthy controls } & \text { Unimpaired LUT function } \\ & \text { No LUTS (3-day bladder diary) } \\ & \text { - No episode of urinary } \\ \text { urgency/week } & - \text { Urinary frequency }<8 / 24 \mathrm{~h} \\ \text { Patients with non-neurogenic } & \text { LUTS }>6 \text { months (3-day } \\ \text { LUTS } & \text { bladder diary) } \\ & -\geq 2 \text { episodes of urinary } \\ & \text { urgency/week } \\ & - \text { Urinary frequency }>8 / 24 \mathrm{~h}\end{array}$

\section{Exclusion criteria}

- Pregnancy or breast feeding

- Any craniocerebral injury or surgery

- Any permanent ferromagnetic implant

- Any previous surgery of LUT/genitalia

- Any anatomical anomaly of LUT/genitalia

- Any LUT malignancy

- PVR > $150 \mathrm{~mL}$

- UTI

- Impaired LUT function

- Any LUTS (3-day bladder diary)

- Any number of episodes of urinary urgency/week

- Urinary frequency $>8 / 24 \mathrm{~h}$

- Any neurological, psychological, metabolic or cardiovascular disease

- Any concomitant treatment for the LUT (eg, neuromodulation)

- SUI

- Indwelling catheters or necessity to perform ISC

ISC, intermittent self-catheterisation; LUTS, lower urinary tract symptoms; PVR, postvoid residual; SUI, stress urinary incontinence. 
Participants of both groups will be matched according to age and gender.

Patients with non-neurogenic LUTS will be recruited from our own department (Neuro-Urology, Balgrist University Hospital, Zürich, Switzerland) and through our partners at the University Hospital Zürich and the Triemli Hospital Zürich. Eligible patients with nonneurogenic LUTS and healthy controls will be invited to a first visit (screening) during which detailed information about the study, in particular the aims, methods, possible risks and side effects, will be given. After obtaining written informed consent, the following data will be collected: medical history, a 3-day bladder diary, urine sample to exclude urinary tract infection (UTI) and pregnancy in female participants, urodynamic parameters and postvoid residual measured by ultrasound as well as standardised questionnaires regarding LUTS and QoL. The validated German versions of these questionnaires will be used with permission of the International Consultation on Incontinence Modular Questionnaire ((ICIQ), Bristol Urological Institute, Southmead Hospital Bristol, UK) and will address the LUTS (ICIQ-LUTS) in women (ICIQ-FLUTS) and men (ICIQ-MLUTS), whereas the ICIQ-LUTSQoL will display the QoL in regard to LUTS.

\section{Determination of sample size}

A power analysis was conducted using G*Power (http:// www.gpower.hhu.de). In order to have sufficient power $(0.80)$ to detect a large effect size (0.80) between healthy controls and patients with non-neurogenic LUTS (significance level 0.05), at least 21 participants per group need to be recruited. To demonstrate posttreatment effects in patients with non-neurogenic LUTS compared with their baseline using the same power, effect size and significance level, at least 12 participants for each treatment option are necessary. These sample sizes are in line with earlier studies ${ }^{22} 23$ including our own, ${ }^{14} 29$ which provided statistical evidence using small collectives, that is, between 12 and 21 participants.

\section{Study location}

- Neuro-Urology, Spinal Cord Injury Centre and Research, University of Zürich, Balgrist University Hospital, Zürich, Switzerland.

- MR-Centre, University Hospital Zürich, Zürich, Switzerland.

\section{Partners}

- Institute of Neuro-Radiology, University of Zürich, University Hospital Zürich, Zürich, Switzerland.

- Departments of Urology and Gynaecology, University Hospital Zürich, Zürich, Switzerland.

- Department of Urology and Gynaecology, Triemli Hospital, Zürich, Switzerland.

\section{Investigations}

Following screening and study inclusion, all participants will be scheduled for the second and third visits (first and second MRI measurements) at the MR-Centre. Patients with non-neurogenic LUTS will return for a fourth visit (third MRI measurement), either after receiving treatment for LUTS or without treatment acting as a direct control group within the patient cohort (figure 1).

All MRI measurements (figure 2) will be performed using a Philips Ingenia 3 T MR scanner (Philips Medical Systems, Best, The Netherlands) with a 16-channel head

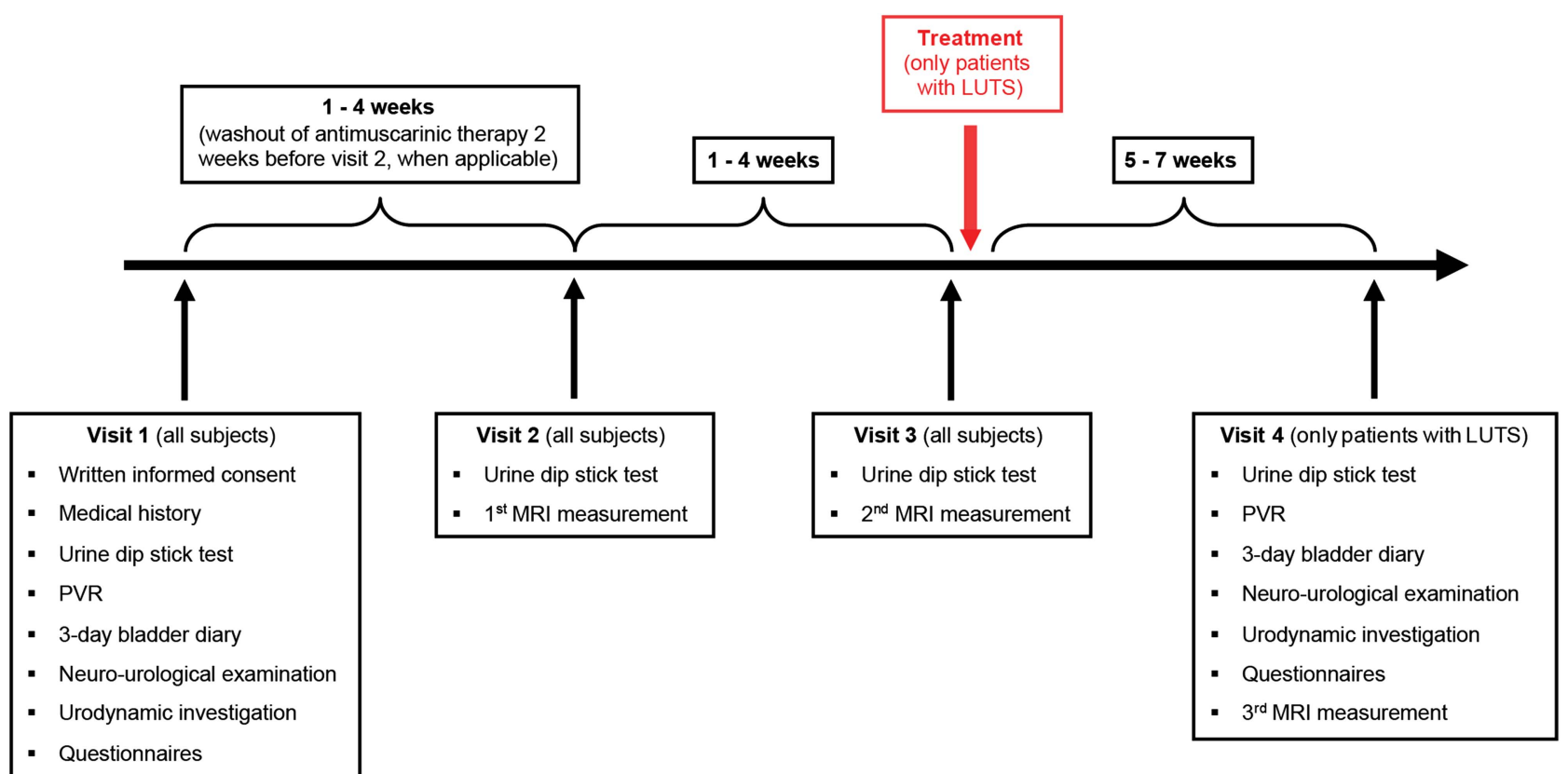

Figure 1 Timetable and characteristics of all four visits. LUTS, lower urinary tract symptoms; MRI, magnetic resonance imaging; PVR, post void residual; QoL, quality of life. 

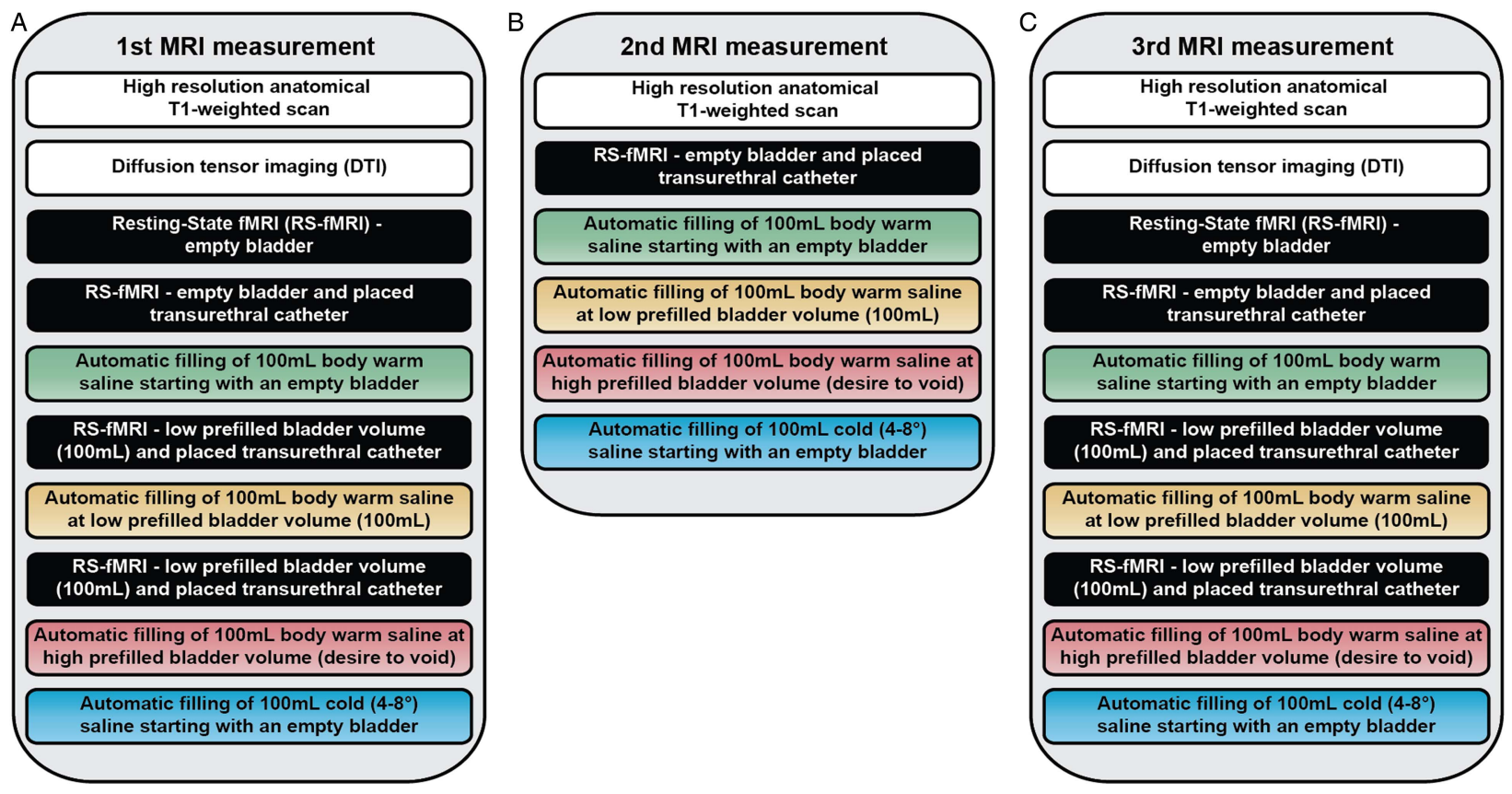

Figure 2 Schematic protocol of operational sequences of MRI measurements including functional MRI (fMRI): (A) first MRI measurement, (B) second MRI measurement and (C) third MRI measurement.

coil. During the second visit, we will acquire the following neuroimaging data. Structural measurements will contain T1-weighted MRI and DTI. Functional measurements will comprise RS-fMRIs and task-related fMRIs. Four different RS-fMRIs will be applied, that is, (1) at baseline with an empty bladder, (2) with an empty bladder plus transurethral catheter, (3) with a low prefilled bladder ( $100 \mathrm{~mL}$ saline, body warm) prior to task-related fMRI and (4) after a task-related fMRI, to understand whether manipulation of sensory perception (induced by catheter or prefilling) will alter the default mode network. ${ }^{20}$ The task-related fMRI will be acquired during four different bladder tasks (figure 3). In order to precisely fill and drain the bladder (ie, specific volume and duration of time), we designed an automated MR-compatible and MR-synchronised pump system. In the first three task-related fMRIs, we will examine the effect of visceral bladder sensation by automated, repetitive filling with $100 \mathrm{~mL}$ body warm saline starting with (1) an empty bladder, (2) a low prefilled bladder and (3) a high prefilled bladder (persistent desire to void). The fourth task-related fMRI will consist of automated, repetitive filling of $100 \mathrm{~mL}$ cold $\left(4-8^{\circ} \mathrm{C}\right)$ saline starting with an empty bladder to investigate the neural correlates of cold bladder sensation (figure 3). Participants will rate their desire to void and their level of pain using a displayed visual analogue scale and an fMRI-compatible handheld response system. ${ }^{30}$

During the second MRI measurement (third visit, 1-4 weeks later), we will utilise a selection of MRI measurements for the purpose of reliability analysis, ${ }^{28}$ that is, RS-fMRI (baseline with an empty bladder plus transurethral catheter) and task-related fMRI to compare within and between groups.
The third MRI measurement will be identical to the first MRI measurement to evaluate post-treatment versus pretreatment effects in patients with non-neurogenic LUTS. The time from the start of treatment to the fourth visit, that is, $5-7$ weeks (figure 1 ), is necessary to let clinical improvements develop. 3132

\section{Safety}

The staff involved in this study will be instructed and trained according to the safety regulations of the MR-Centre of the University Hospital Zürich. All participants will be asked to remove any ferromagnetic items, for example, bra, earrings, chains, rings and piercings prior to entering the scanner room. All participants will be provided with standardised clinical scrubs instead of wearing their own clothes. Before every MRI measurement (visits 2, 3 and 4), urine samples will be analysed from every participant in order to exclude UTI or pregnancy. In case of pregnancy, the participant will be excluded from the study and referred to a gynaecologist. In case of UTI, the participant will not undergo the experiment, but will receive immediate antibiotic treatment if the UTI is symptomatic or be treated depending on further microbiological urine analysis in the absence of UTI symptoms. The participant can be reassigned to the study if the microbiological urine analysis shows no evidence of a UTI or the UTI has been successfully treated.

In the situation of an adverse event (AE) or a severe $\mathrm{AE}$ (SAE), as defined by the International Conference on Harmonisation (ICH) Good Clinical Practice (GCP) Guidelines (E6) ${ }^{33}$ and International Organization for Standardization (ISO, 14155),$^{34}$ appropriate actions will be executed and the according body (principle investigator, 
A) 1st task-realted fMRI - Automated, repetitive bladder filling with $100 \mathrm{~mL}$ body warm saline starting with an empty bladder.

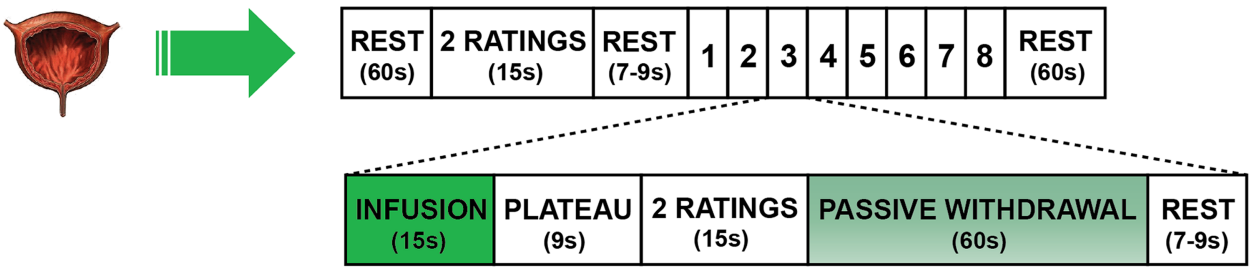

B) 2nd task-realted fMRI - Automated, repetitive bladder filling with $100 \mathrm{~mL}$ body warm saline starting with a low prefilled bladder volume (100mL).

\begin{tabular}{|c|c|c|c|c|c|c|c|c|c|c|c|}
\hline & & $\begin{array}{l}\text { REST } \\
\text { (60s) }\end{array}$ & \multicolumn{2}{|c|}{$\begin{array}{c}2 \text { RATINGS } \\
\text { (15s) }\end{array}$} & $\begin{array}{l}\text { REST } \\
(7-9 s)\end{array}$ & 12 & 23 & \begin{tabular}{l|l|}
4 & 5
\end{tabular} & \begin{tabular}{|l|l|}
6 & 7 \\
\end{tabular} & $8 \mid \begin{array}{c}\text { REST } \\
(60 \mathrm{~s})\end{array}$ & \\
\hline $\begin{array}{c}\text { INFUSION } \\
(15 s)\end{array}$ & \begin{tabular}{|} 
PLATEAU \\
$(9 \mathrm{~s})$
\end{tabular} & $\begin{array}{r}2 \mathrm{RA} \\
\text { (15 }\end{array}$ & $\begin{array}{l}\text { TINGS } \\
5 \mathrm{~s})\end{array}$ & $\begin{array}{l}\text { RES } \\
(7-9 s\end{array}$ & \begin{tabular}{l|l} 
T) & WITH
\end{tabular} & $\begin{array}{r}\text { HDRA } \\
(15 s)\end{array}$ & s) & PLA & $\begin{array}{l}\text { ATEAU } \\
(9 \mathrm{~s})\end{array}$ & $\begin{array}{c}2 \text { RATINGS } \\
(15 \mathrm{~s})\end{array}$ & $\begin{array}{l}\text { REST } \\
\text { (7-9s) }\end{array}$ \\
\hline
\end{tabular}

C) 3rd task-realted fMRI - Automated, repetitive bladder filling with $100 \mathrm{~mL}$ body warm saline starting with a high prefilled bladder volume (desire to void).

\begin{tabular}{|c|c|c|c|c|c|c|c|c|c|c|c|c|c|c|c|}
\hline & & $\begin{array}{c}\text { REST } \\
(60 \mathrm{~s})\end{array}$ & \multicolumn{2}{|c|}{$\begin{array}{c}2 \text { RATINGS } \\
(15 \mathrm{~s})\end{array}$} & \multicolumn{3}{|c|}{\begin{tabular}{r|r} 
REST & 1 \\
$(7-9 s)$ &
\end{tabular}} & 2 & \begin{tabular}{l|l}
3 & 4
\end{tabular} & 5 & 6 & \multicolumn{2}{|c|}{\begin{tabular}{l|l}
7 & 8
\end{tabular}} & $\begin{array}{c}\text { REST } \\
(60 \mathrm{~s})\end{array}$ & \\
\hline $\begin{array}{l}\text { NFUSION } \\
(15 \mathrm{~s})\end{array}$ & $\begin{array}{c}\text { PLATEAU } \\
\text { (9s) }\end{array}$ & $\begin{array}{r}2 \mathrm{RA} \\
(1\end{array}$ & $\begin{array}{l}\text { TINGS } \\
\text { 5s) }\end{array}$ & $\begin{array}{l}\text { RES } \\
(7-9 \mathrm{~s}\end{array}$ & & WITr & $\begin{array}{l}\text { HDR } \\
(15\end{array}$ & $\begin{array}{l}\text { RAV } \\
\text { is) }\end{array}$ & WAL & & $\begin{array}{l}\text { LAT } \\
\text { (9s }\end{array}$ & EAU & & $\begin{array}{l}\text { RATINGS } \\
\text { (15s) }\end{array}$ & $\begin{array}{l}\text { REST } \\
\text { (7-9s) }\end{array}$ \\
\hline
\end{tabular}

D) 4 th task-realted fMRI - Automated, repetitive bladder filling with $100 \mathrm{~mL}$ cold $\left(4-8^{\circ} \mathrm{C}\right)$ saline starting with an empty bladder.

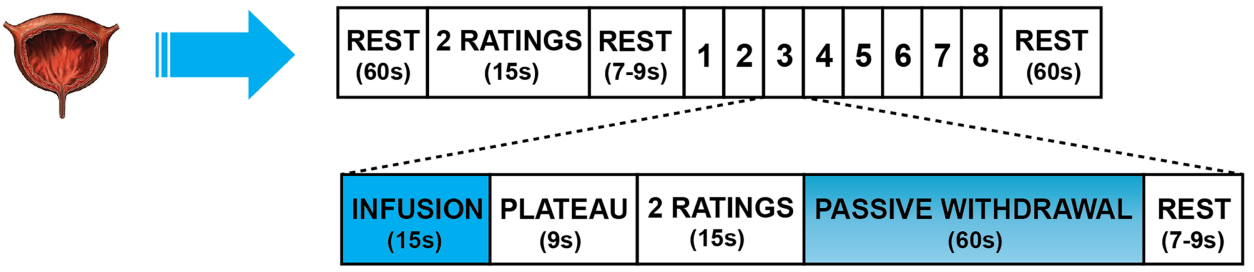

Figure 3 BOLD signal intensity changes during task-related fMRI in relation to the specific condition, that is, infusion or to a contrast, that is, low versus full bladder volume, during two (healthy controls) or three (patients with non-neurogenic LUTS) visits. Investigation of these changes will focus on supraspinal regions of interest (ROI) that are known from the existing literature, for example, pons, insula, anterior cingulate cortex, thalamus, hypothalamus, supplementary motor area and prefrontal cortex. However, the precise selection of ROls will be based on the coordinates of the peak activations during task-related fMRI taken from the Montreal Neurological Institute (MNI) space.

ethics committee) will be informed. All AEs and SAEs will be followed as long as medically indicated.

\section{Study outcome measures}

Primary

A. BOLD signal intensity changes in a priori supraspinal regions of interest (ROI), for example, pons, insula, anterior cingulate cortex, thalamus, hypothalamus, supplementary motor area and prefrontal cortex, during task-related fMRI in relation to the specific condition, that is, infusion or to a contrast, that is, low versus full bladder volume, during two (healthy controls) or three (patients with non-neurogenic LUTS) visits. The precise selection of ROIs will be based on the coordinates of the peak activations during task-related fMRI taken from the Montreal Neurological Institute (MNI) space.

B. Reliability of BOLD signal changes during RS-fMRI and task-related fMRI across visits (eg, second and third visits) in healthy controls and patients with non-neurogenic LUTS.

C. BOLD signal changes in supraspinal ROIs during task-related fMRI in patients with non-neurogenic LUTS before and after treatment to quantify the link between BOLD signal changes and treatment efficacy.

\section{Secondary}

A. Structural differences between healthy controls and patients with non-neurogenic LUTS, and changes in the post-treatment versus pretreatment state of grey matter concentration using VBM. 
B. Structural connectivity (SC) and FC between supraspinal ROIs to identify specific alterations with $\mathrm{DTI}^{35}$ between healthy controls and patients with nonneurogenic LUTS (including post-treatment versus pretreatment changes). This will include whole-brain fractional anisotropy (FA) and mean diffusivity (MD) comparison as well as probabilistic tractography between ROIs (white matter fibre structure).

C. Differences in BOLD signals during RS-fMRI between healthy controls and patients with nonneurogenic LUTS (including post-treatment versus pretreatment changes) in regard to intrahemispheric and interhemispheric connectivity. ${ }^{36}$ Investigation will focus on whether these signals differ (1) already at baseline or are influenced by the (2) presence of a catheter, (3) by prefilling and/or (4) by taskrelated fMRI.

D. Clinical scores (eg, bladder volume, urodynamic parameters and level of desire to void during fMRI) will be correlated to BOLD signal changes as well as to structural markers (eg, grey matter volume or number of white matter tracts between ROIs) using regression analyses.

\section{Data analysis}

Clinical data, for example, urodynamic parameters, 3-day bladder diary outcome and questionnaire scores, will be statistically analysed and compared between groups using IBM's Statistical Package for the Social Sciences (SPSS) V.19.0 or newer (Armonk, New York, USA) and will be presented with means and SDs or with medians and IQRs as appropriate.

The neuroimaging data will be analysed using statistical parametric mapping (SPM) V.8 or newer (Wellcome Department of Imaging Neuroscience, University College London, UK). Preprocessing of functional data from each task-related fMRI will be carried out for each participant individually. The images will be realigned to the first scan, unwarped to control for movement-induced and susceptibility-induced image distortions, ${ }^{37}$ spatially coregistered to the T1-weighted image and normalised to the MNI anatomical standard space. At last, the functional data will be smoothed spatially with an isotropic Gaussian kernel. Thereafter, first-level analysis using the general linear model will be performed to create contrasts of interest, for example, low versus full bladder or infusion versus withdrawal. ${ }^{38}$ The six movement parameters will be modelled as additional regressors to control for potential head motion.

Second-level factorial design will include at least (1) one-sample t test to compute a mean for each group, (2) two-sample $\mathrm{t}$ tests to compare healthy controls and patients with non-neurogenic LUTS and (3) paired t tests to evaluate post-treatment versus pretreatment effects in patients with non-neurogenic LUTS. The ICC for task-related and RS-fMRI reliability will be analysed using the SPM-compatible ICC toolbox (http://www.kcl.ac.uk/ iop/depts/neuroimaging/research/imaginganalysis/ Software/ICC-Toolbox.aspx).
Association of individual (clinical) variables with BOLD signal changes will be assessed by whole-brain and ROI-based correlation analyses (correlation coefficients will be reported).

DTI data will be analysed using TBSS (http://fsl. fmrib.ox.ac.uk/fsl/fsl4.0/tbss/index) and BrainVoyager (http://www.brainvoyager.com/downloads/downloads. html) with the following established DTI analyses: whole-brain FA and MD comparison between groups as well as probabilistic tractography between ROIs. Seed and target regions, that is, ROIs will be defined (1) a priori using anatomic coordinates, for example, from the SPM toolbox WFU_PickAtlas (http://fmri. wfubmc.edu/software/PickAtlas) and (2) from peak activations acquired from task-related fMRI and RS-fMRI on the standard MNI space. Volumetric changes in grey and white matter will be analysed using VBM, for example, the VBM toolbox (http:// dbm.neuro.uni-jena.de/vbm) in SPM.

\section{ETHICS AND DISSEMINATION}

This cohort study will be performed in accordance with the World Medical Association Declaration of Helsinki ${ }^{39}$ and the guidelines of the Swiss Academy of Medical Sciences. ${ }^{40}$

Furthermore, handling of all personal data will strictly comply with the federal law of data protection in Switzerland. ${ }^{41}$

This study has been registered at clinicaltrials.gov (http://www.clinicaltrials.gov/ct2/show/NCT01768910).

\section{DISCUSSION}

This study will investigate supraspinal LUT control in healthy controls and patients with non-neurogenic LUTS using a multimodal imaging protocol, that is, structural (T1-weighted and DTI) and fMRI (RS-fMRI and task-related fMRI), to examine haemodynamic responses to LUT stimulation. From the acquired neuroimaging data, SC and FC, and structural integrity evaluation of white matter tracts and grey matter concentration (using VBM) will be analysed to identify specific alterations of the supraspinal LUT control. In addition, effects on the supraspinal LUT control after treatment for LUTS (eg, antimuscarinergics or botulinum toxin) will be investigated. It is expected that this study will provide new insights into the supraspinal neuronal mechanisms and networks responsible for LUT control. The findings will help to verify, amend or adjust neuronal circuitry models established from findings in healthy controls, now in the context of patients with nonneurogenic LUTS. The use of newer imaging and evaluation techniques has the potential to serve as quantifiable outcome measures for therapy success and provide evidence for non-responders of LUTS treatment.

\section{Author affiliations}

${ }^{1}$ Department of Neuro-Urology, Spinal Cord Injury Centre \& Research, University of Zürich, Balgrist University Hospital, Zürich, Switzerland 
${ }^{2}$ Institute of Neuro-Radiology, University of Zürich, University Hospital Zürich, Zürich, Switzerland

${ }^{3}$ Department of Urology, University Hospital Maastricht, Maastricht, The Netherlands

Correction notice Following online publication of this article it has been noted that errors were mistakenly introduced. Figure 3 legend was inadvertently transposed with a section of the main text. The correct figure 3 legend is as follows:

Figure 3: Schematic diagram of the scan paradigm of four different task-related functional MRIs (fMRIs) at visits 2, 3 and 4. All task-related fMRIs identically start with a 'baseline' rest (60 s, no specific stimulus or task is performed), a 'baseline' rating of desire to void and level of pain, a short rest jittered between 7 and $9 \mathrm{~s}$ in which blood-oxygen-level dependent (BOLD) activation resulting from motor activity during the previous rating will return to baseline to avoid contamination of the following condition and conclude with a 'last' rest $60 \mathrm{~s}$, no specific stimulus or task is performed). All task-related fMRIs consist of eight repetitive blocks, each with either five (first and fourth fMRIs) or eight (second and third fMRIs) conditions. (A) Conditions of the first task-related fMRI: (1) automated infusion of $100 \mathrm{~mL}$ body warm saline, (2) plateau phase (bladder distention after infusion is perceived), (3) rating of desire to void and level of pain, (4) passive withdrawal to empty the bladder completely and (5) short rest jittered between 7 and $9 \mathrm{~s}$. This task-related fMRI starts with an empty bladder and will be performed in patients with non-neurogenic LUTS in visits 2, 3 and 4, while in healthy controls only at visit 3 (second MRI measurement). (B and C) Conditions of the second and third task-related fMRIs: (1) automated infusion of $100 \mathrm{~mL}$ warm saline, (2) plateau phase (bladder distention after infusion is perceived), (3) rating of desire to void and level of pain, (4) short rest jittered between 7 and $9 \mathrm{~s}$ in which BOLD activation resulting from motor activity during the previous rating will return to baseline to avoid contamination of the following condition, (5) automated withdrawal of $100 \mathrm{~mL}$, (6) plateau phase (bladder distention after withdrawal is perceived), (7) rating of desire to void and level of pain and (8) short rest jittered between 7 and $9 \mathrm{~s}$ in which BOLD activation resulting from motor activity during the previous rating will return to baseline to avoid contamination of the following condition. The second task-related fMRI (B) starts with a low prefilled bladder volume $(100 \mathrm{~mL})$ and will be performed only in healthy controls at visits 2 and 3 (first and second MRI measurements). The third task-related fMRI (C) starts with a high prefilled bladder volume (persistent desire to void) and will be performed in all participants (patients with non-neurogenic LUTS and healthy controls) during visits 2 and 3 (first and second MRI measurements). Additionally, this task-related fMRI will be carried out in patients with nonneurogenic LUTS at visit 4 (third MRI measurement). (D) Conditions of the fourth task-related fMRI task: (1) automated infusion of $100 \mathrm{~mL}$ cold $\left(4-8^{\circ} \mathrm{C}\right)$ saline, (2) plateau phase (bladder distention after infusion is perceived), (3) rating of desire to void and level of pain, (4) passive withdrawal to empty the bladder completely and (5) short rest jittered between 7 and $9 \mathrm{~s}$. This taskrelated fMRI starts with an empty bladder and will be performed in all participants (patient with non-neurogenic LUTS and healthy controls) during visit 2 and 3 (first and second MRI measurement). Additionally, this taskrelated fMRI will be executed in patients with non-neurogenic LUTS at visit 4 (third MRI measurement).

In addition, on page 5 'Study outcome measures' / 'Primary' the first section

'(A)' should read:

BOLD signal intensity changes during task-related fMRI in relation to the specific condition, that is, infusion or to a contrast, that is, low versus full bladder volume, during two (healthy controls) or three (patients with nonneurogenic LUTS) visits. Investigation of these changes will focus on supraspinal regions of interest (ROI) that are known from the existing literature, for example, pons, insula, anterior cingulate cortex, thalamus, hypothalamus, supplementary motor area and prefrontal cortex. However, the precise selection of ROls will be based on the coordinates of the peak activations during taskrelated fMRI taken from the Montreal Neurological Institute (MNI) space. Finally, in table 1 the expansion of the acronym 'UTI' should have been included - 'UTI, urinary tract infection'.

Acknowledgements The authors would like to acknowledge the Swiss National Science Foundation, SwissLife Jubiläumsstiftung and Swiss Continence Foundation for financial support. Furthermore, they would like to thank the partners contributing to this study: Departments of Urology and
Gynaecology of the University Hospital Zürich and Departments of Urology and Gynaecology, Triemli Hospital, Zürich, Switzerland. They also thank Behnaz Jarrahi for granting figure contents.

Contributors All authors participated in creating the study design. MW and UM drafted the manuscript. LM, SK, PEvK and TMK critically reviewed the manuscript. UM, SK and TMK obtained the funding of this study. All the authors read and approved the final manuscript.

Funding Swiss National Science Foundation (grant number: 135774), SwissLife Jubiläumsstiftung, Swiss Continence Foundation.

Competing interests None.

Ethics approval This study has been approved by the local ethics committee (Kantonale Ethikkommission Zürich, KEK-ZH-Nr. 2011-0346).

Provenance and peer review Not commissioned; externally peer reviewed.

Open Access This is an Open Access article distributed in accordance with the Creative Commons Attribution Non Commercial (CC BY-NC 3.0) license, which permits others to distribute, remix, adapt, build upon this work noncommercially, and license their derivative works on different terms, provided the original work is properly cited and the use is non-commercial. See: http:// creativecommons.org/licenses/by-nc/3.0/

\section{REFERENCES}

1. Irwin DE, Kopp ZS, Agatep B, et al. Worldwide prevalence estimates of lower urinary tract symptoms, overactive bladder, urinary incontinence and bladder outlet obstruction. BJU Int 2011;108:1132-8

2. Irwin DE, Milsom I, Hunskaar S, et al. Population-based survey of urinary incontinence, overactive bladder, and other lower urinary tract symptoms in five countries: results of the EPIC study. Eur Urol 2006;50:1306-14; discussion 14-5.

3. Ganz ML, Smalarz AM, Krupski TL, et al. Economic costs of overactive bladder in the United States. Urology 2010;75:526-32, 32 e1-18.

4. Klotz T, Bruggenjurgen B, Burkart M, et al. The economic costs of overactive bladder in Germany. Eur Urol 2007;51:1654-62; discussion 62-3.

5. Hampel C, Gillitzer R, Pahernik S, et al. Epidemiology and etiology of overactive bladder. Urologe A 2003;42:776-86.

6. Coyne KS, Sexton CC, Irwin DE, et al. The impact of overactive bladder, incontinence and other lower urinary tract symptoms on quality of life, work productivity, sexuality and emotional well-being in men and women: results from the EPIC study. BJU Int 2008;101:1388-95.

7. Irwin DE, Milsom I, Kopp Z, et al. Impact of overactive bladder symptoms on employment, social interactions and emotional well-being in six European countries. BJU Int 2006;97:96-100.

8. Blok BF. Brain control of the lower urinary tract. Scand J Urol Nephrol Suppl 2002:11-15.

9. Fowler CJ, Griffiths D, de Groat WC. The neural control of micturition. Nat Rev Neurosci 2008;9:453-66.

10. Fowler CJ, Griffiths DJ. A decade of functional brain imaging applied to bladder control. Neurourol Urodyn 2010;29:49-55.

11. Herzog J, Weiss $\mathrm{PH}$, Assmus $\mathrm{A}$, et al. Subthalamic stimulation modulates cortical control of urinary bladder in Parkinson's disease. Brain 2006;129(Pt 12):3366-75.

12. Herzog J, Weiss $\mathrm{PH}$, Assmus A, et al. Improved sensory gating of urinary bladder afferents in Parkinson's disease following subthalamic stimulation. Brain 2008;131(Pt 1):132-45.

13. Kitta $\mathrm{T}$, Kakizaki $\mathrm{H}$, Furuno $\mathrm{T}$, et al. Brain activation during detrusor overactivity in patients with Parkinson's disease: a positron emission tomography study. J Urol 2006;175(3 Pt 1):994-8.

14. Mehnert U, Michels L, Zempleni MZ, et al. The supraspinal neural correlate of bladder cold sensation-an fMRI study. Hum Brain Mapp 2011;32:835-45.

15. Biswal BB, Van Kylen J, Hyde JS. Simultaneous assessment of flow and BOLD signals in resting-state functional connectivity maps. NMR Biomed 1997;10:165-70.

16. Cauda F, Costa T, Torta DM, et al. Meta-analytic clustering of the insular cortex: characterizing the meta-analytic connectivity of the insula when involved in active tasks. Neuroimage 2012;62:343-55

17. Greicius MD, Supekar K, Menon V, et al. Resting-state functional connectivity reflects structural connectivity in the default mode network. Cereb Cortex 2009;19:72-8.

18. Gusnard DA, Akbudak E, Shulman GL, et al. Medial prefrontal cortex and self-referential mental activity: relation to a default mode of brain function. Proc Natl Acad Sci USA 2001;98:4259-64. 
19. Gusnard DA, Raichle ME. Searching for a baseline: functional imaging and the resting human brain. Nat Rev Neurosci 2001;2:685-94.

20. Raichle ME, MacLeod AM, Snyder AZ, et al. A default mode of brain function. Proc Natl Acad Sci USA 2001;98:676-82.

21. Basser PJ, Mattiello J, LeBihan D. MR diffusion tensor spectroscopy and imaging. Biophys J 1994;66:259-67.

22. Théaudin M, Saliou G, Denier C, et al. A correlation between fractional anisotropy variations and clinical recovery in spinal cord infarctions. J Neuroimaging 2013;23:256-8.

23. van der Jagt PK, Dik P, Froeling $M$, et al. Architectural configuration and microstructural properties of the sacral plexus: a diffusion tensor MRI and fiber tractography study. Neuroimage 2012;62:1792-9.

24. Andrew J, Nathan PW. Lesions on the anterior frontal lobes and disturbances of micturition and defaecation. Brain 1964;87:233-62.

25. Holstege G. Micturition and the soul. J Comp Neurol 2005;493:15-20.

26. de Groat WC. A neurologic basis for the overactive bladder. Urology 1997;50(6A Suppl):36-52; discussion 53-6.

27. Ashburner J, Friston KJ. Voxel-based morphometry-the methods. Neuroimage 2000;11(6 Pt 1):805-21.

28. Caceres A, Hall DL, Zelaya FO, et al. Measuring fMRI reliability with the intra-class correlation coefficient. Neuroimage 2009;45:758-68.

29. Michels L, Mehnert U, Boy S, et al The somatosensory representation of the human clitoris: an fMRI study. Neuroimage 2010;49:177-84.

30. Jarrahi B, Wanek J, Mehnert U, et al. An fMRI-compatible multi-configurable handheld response system using an intensity-modulated fiber-optic sensor. Conf Proc IEEE Eng Med Biol Soc 2013;2013:6349-52.

31. Agency for Healthcare Research and Quality. Treatment of Overactive Bladder in Women, 2009.

32. Gormley EA, Lightner DJ, Burgio KL, et al. Diagnosis and treatment of overactive bladder (non-neurogenic) in adults: AUA/SUFU guideline. J Urol 2012;188(6 Suppl):2455-63.

33. International conference on harmonisation. Good clinical practice guideline, 1996

34. International organization for standardization. ISO 14155, 2011.

35. Kucyi A, Moayedi M, Weissman-Fogel I, et al. Hemispheric asymmetry in white matter connectivity of the temporoparietal junction with the insula and prefrontal cortex. PLOS ONE 2012;7: e35589.

36. Maldjian JA, Davenport EM, Whitlow CT. Graph theoretical analysis of resting-state MEG data: identifying interhemispheric connectivity and the default mode. Neuroimage 2014;96:88-94.

37. Andersson JL, Hutton C, Ashburner J, et al. Modeling geometric deformations in EPI time series. Neuroimage 2001;13:903-19.

38. Friston $\mathrm{KJ}$, Holmes AP, Poline JB, et al. Analysis of $\mathrm{AMRI}$ time-series revisited. Neuroimage 1995;2:45-53.

39. World Medical Association. Declaration of Helsinki-Ethical principles for medical research involving human subjects, 1964

40. Swiss Academy of Medical Sciences. Guideline-Concerning scientific research involving human beings, 2009.

41. The Federal Authorities of the Swiss Confederation. Bundesgesetz über den Datenschutz (DSG) vom 19. Juni 1992, Stand. 01.01.2011, 1992. 


\section{Correction}

Walter M, Michels L, Kollias S, et al. Protocol for a prospective neuroimaging study investigating the supraspinal control of lower urinary tract function in healthy controls and patients with non-neurogenic lower urinary tract symptoms. BMJ Open 2014;4:e004357.

Figure 3 legend was inadvertently transposed with a section of the main text. The correct figure 3 legend is as follows:

Figure 3: Schematic diagram of the scan paradigm of four different task-related functional MRIs (fMRIs) at visits 2, 3 and 4. All task-related fMRIs identically start with a 'baseline' rest ( $60 \mathrm{~s}$, no specific stimulus or task is performed), a 'baseline' rating of desire to void and level of pain, a short rest jittered between 7 and $9 \mathrm{~s}$ in which blood-oxygen-level dependent (BOLD) activation resulting from motor activity during the previous rating will return to baseline to avoid contamination of the following condition and conclude with a 'last' rest $(60 \mathrm{~s}$, no specific stimulus or task is performed). All task-related fMRIs consist of eight repetitive blocks, each with either five (first and fourth fMRIs) or eight (second and third fMRIs) conditions. (A) Conditions of the first task-related fMRI: (1) automated infusion of $100 \mathrm{~mL}$ body warm saline, (2) plateau phase (bladder distention after infusion is perceived), (3) rating of desire to void and level of pain, (4) passive withdrawal to empty the bladder completely and (5) short rest jittered between 7 and $9 \mathrm{~s}$. This task-related fMRI starts with an empty bladder and will be performed in patients with non-neurogenic LUTS in visits 2, 3 and 4, while in healthy controls only at visit 3 (second MRI measurement). (B and C) Conditions of the second and third task-related fMRIs: (1) automated infusion of $100 \mathrm{~mL}$ warm saline, (2) plateau phase (bladder distention after infusion is perceived), (3) rating of desire to void and level of pain, (4) short rest jittered between 7 and $9 \mathrm{~s}$ in which BOLD activation resulting from motor activity during the previous rating will return to baseline to avoid contamination of the following condition, (5) automated withdrawal of $100 \mathrm{~mL}$, (6) plateau phase (bladder distention after withdrawal is perceived), (7) rating of desire to void and level of pain and (8) short rest jittered between 7 and $9 \mathrm{~s}$ in which BOLD activation resulting from motor activity during the previous rating will return to baseline to avoid contamination of the following condition. The second task-related fMRI (B) starts with a low prefilled bladder volume $(100 \mathrm{~mL})$ and will be performed only in healthy controls at visits 2 and 3 (first and second MRI measurements). The third task-related fMRI (C) starts with a high prefilled bladder volume (persistent desire to void) and will be performed in all participants (patients with non-neurogenic LUTS and healthy controls) during visits 2 and 3 (first and second MRI measurements). Additionally, this task-related fMRI will be carried out in patients with non-neurogenic LUTS at visit 4 (third MRI measurement). (D) Conditions of the fourth task-related fMRI task: (1) automated infusion of $100 \mathrm{~mL}$ cold $\left(4-8^{\circ} \mathrm{C}\right)$ saline, (2) plateau phase (bladder distention after infusion is perceived), (3) rating of desire to void and level of pain, (4) passive withdrawal to empty the bladder completely and (5) short rest jittered between 7 and $9 \mathrm{~s}$. This task-related fMRI starts with an empty bladder and will be performed in all participants (patient with non-neurogenic LUTS and healthy controls) during visit 2 and 3 (first and second MRI measurement). Additionally, this task-related fMRI will be executed in patients with non-neurogenic LUTS at visit 4 (third MRI measurement).

In addition, on page 5 'Study outcome measures'/'Primary' the first section '(A)' should read:

BOLD signal intensity changes during task-related fMRI in relation to the specific condition, that is, infusion or to a contrast, that is, low versus full bladder volume, during two (healthy controls) or three (patients with non-neurogenic LUTS) visits. Investigation of these changes will focus on supraspinal regions of interest (ROI) that are known from the existing literature, for example, pons, insula, anterior cingulate cortex, thalamus, hypothalamus, supplementary motor area and prefrontal cortex. However, the precise selection of ROIs will be based on the coordinates of the peak activations during task-related fMRI taken from the Montreal Neurological Institute (MNI) space.

Finally, in table 1 the expansion of the acronym 'UTI' should have been included - 'UTI, urinary tract infection'.

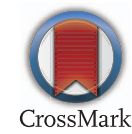

